OPEN ACCESS

Edited by:

Jie Sun,

Wenzhou Medical University, China

Reviewed by:

Chong Tang,

Beijing Genomics Institute (BGI), China

Ming Li,

Beijing Center for Disease Prevention and Control (Beijing CDC), China

*Correspondence:

Yongqiang $\mathrm{HaO}$

Haoyq1664@sh9hospital.org.cn

Specialty section: This article was submitted to

Cancer Genetics,

a section of the journal

Frontiers in Oncology

Received: 03 April 2021

Accepted: 29 April 2021

Published: 11 June 2021

Citation:

Pang B and Hao Y (2021) Integrated Analysis of the Transcriptome Profile

Reveals the Potential Roles Played by

Long Noncoding RNAs in Immunotherapy for Sarcoma.

Front. Oncol. 11:690486. doi: $10.3389 /$ fonc. 2021.690486

\section{Integrated Analysis of the Transcriptome Profile Reveals the Potential Roles Played by Long Noncoding RNAs in Immunotherapy for Sarcoma}

\author{
Boran Pang ${ }^{1,2}$ and Yongqiang Hao ${ }^{1,2^{*}}$ \\ ${ }^{1}$ Shanghai Key Laboratory of Orthopaedic Implants, Department of Orthopaedic Surgery, Shanghai Ninth People's Hospital, \\ Shanghai Jiao Tong University School of Medicine, Shanghai, China, ${ }^{2}$ Clinical and Translational Research Center for 3D \\ Printing Technology, Shanghai Ninth People's Hospital, Shanghai Jiao Tong University School of Medicine, Shanghai, China
}

Background: Long-term survival is still low for high-risk patients with soft tissue sarcoma treated with standard management options, including surgery, radiation, and chemotherapy. Immunotherapy is a promising new potential treatment paradigm. However, the application of immune checkpoint inhibitors for the treatment of patients with sarcoma did not yield promising results in a clinical trial. Therefore, there is a considerable need to identify factors that may lead to immune checkpoint inhibitor resistance.

Methods: In this study, we performed a bioinformatic analysis of The Cancer Genome Atlas (TCGA) to detect key long noncoding RNAs (IncRNAs) that were correlated with immune checkpoint inhibitory molecules in sarcoma. The expression levels of these IncRNAs and their correlation with patient prognosis were explored. The upstream long noncoding RNAs were also examined via 450K array data from the TCGA. The potential roles of these IncRNAs were further examined via KEGG and GO analysis using DAVID online software. Finally, the relationship between these IncRNAs and immune cell infiltration in tumors and their effect on immune checkpoint inhibitors were further explored.

Results: We identified IncRNAs correlated with tumor cell immune evasion in sarcoma. The expression of these IncRNAs was upregulated and correlated with worse prognosis in sarcoma and other human cancer types. Moreover, low DNA methylation occupation of these IncRNA loci was detected. Negative correlations between DNA methylation and IncRNA expression were also found in sarcoma and other human cancer types. KEGG and GO analyses indicated that these IncRNAs correlated with immune evasion and negative regulation of the immune response in sarcoma. Finally, high expression of these IncRNAs correlated with more suppressive immune cell infiltration and reduced sensitivity to immune checkpoint inhibitors in sarcoma and other human cancer types. 


\begin{abstract}
Conclusion: Our results suggest that long noncoding RNAs confer immune checkpoint inhibitor resistance in human cancer. Further characterization of these IncRNAs may help to elucidate the mechanisms underlying immune checkpoint inhibitor resistance and uncover a novel therapeutic intervention point for immunotherapy.
\end{abstract}

Keywords: long noncoding RNA (IncRNA), immunotherapy, immune checkpoint inhibitor, resistance, sarcoma

\section{INTRODUCTION}

Sarcomas represent a cohort of rare and heterogeneous tumors with over 100 different histological subtypes occurring predominantly in the trunk, extremity, and retroperitoneal areas (1). These tumors exhibit a wide range of differing behaviors and underlying molecular pathologies (2-4). Recently, subtype-specific cancer biology has revealed distinct molecular alterations responsible for tumor initiation and progression. The rarity and molecular heterogeneity of sarcoma creates challenges for the development of targeted therapeutics. Fortunately, in contrast to the genomic landscape of other tumor types, the genomic landscape of sarcomas is relatively simple, which offers the opportunity to identify driver events and translate these discoveries into clinically useful biomarkers $(5,6)$. This may improve the understanding of sarcoma biology, accelerating clinical translational research.

Multidisciplinary management is best for sarcoma therapy (7-12). Surgical resection with or without radiotherapy and chemotherapy is the standard therapy for localized sarcoma (10, 13). Systemic therapy has a prominent role in the multidisciplinary management of locally inoperable or metastatic sarcomas $(7,14)$. Cytotoxic chemotherapy has been the mainstay therapy for treating advanced-stage disease, with overall response rates of approximately $25 \%$ in the first-line setting. In patients with unresectable and metastatic disease, the standard treatment is a single-agent protocol administered with palliative intent. Despite the use of chemotherapy, advanced-stage sarcoma is almost invariably fatal. Thus, it is of urgent importance to develop methods to identify the patients most likely to benefit from current treatments and to develop novel therapeutic strategies. A number of therapeutic targets for sarcoma have been developed with recent scientific technological advancements, including SRC inhibitors, MET and ALK inhibitors, histone deacetylase inhibitors, MDM2 inhibitors and poly (ADP-ribose) polymerase (PARP) inhibitors (15-21). However, these inhibitors yielded unsatisfactory results in clinical trials.

Immunotherapy that has been used for melanoma is a new potential therapy paradigm that holds great promise for sarcoma treatment (22-26). Immune checkpoint molecules target the interface between antigen-presenting cells and $\mathrm{T}$ cells and require the clustering of $\mathrm{T}$ cells and costimulatory receptors. There are negative-feedback mechanisms to prevent overstimulation, which can be subverted to suppress the immune system in cancer, such as the upregulation of CTLA-4 expression. A significant advance in the understanding of sarcoma immune biology is required as it has recently been shown that the monoclonal antibodies ipilimumab and nivolumab are successful for the treatment of melanoma. The inhibition of CTLA-4 expression with the monoclonal antibody ipilimumab reverses immune suppression and promotes a significant anti-cancer response in melanoma. However, while ipilimumab given to patients with synovial sarcoma was well tolerated, no obvious efficacy was observed (27). Therefore, the identification of potential factors leading to immune checkpoint inhibitor resistance is urgently needed.

\section{MATERIALS AND METHODS}

\section{Expression Data and Clinical Data Acquisition}

The gene expression profiles and clinical information of patients were obtained from The Cancer Genome Atlas (TCGA) (https:// cancergenome.nih.gov/). The clinical data included survival time, survival status, sex, age, TNM stage, pathological stage and so on.

\section{Identification of Immune Checkpoint Related LncRNA}

Immune checkpoint-related lncRNAs were identified through Pearson correlation with immune checkpoint inhibitory molecules (CD80, CD86, CD274, PDCD1 LG2 and LGALS9) ( $\mathrm{R}>0.3$ and $\mathrm{P}<1.00 \mathrm{E}-8)$ according to previous studies (28). The results were analyzed online via Venny 2.1.0 software (https:// bioinfogp.cnb.csic.es/tools/venny/). P $<0.05$ indicated statistical significance.

\section{Analysis of Prognosis}

Overall survival (OS) and progression-free interval (PFI) were defined as the time from treatment until the occurrence of death or progression, respectively. The log-rank test was used to examine the survival difference between different patient groups. $\mathrm{P}<0.05$ indicated statistical significance.

\section{Guilt-by-Association Analysis and Functional Enrichment Analysis}

Guilt-by-association analysis was performed to identify immune checkpoint-related lncRNAs that are positively correlated with the target genes according to a previous study (29-32). Pairwise Pearson correlation was performed between the expression of immune checkpoint inhibitory molecule-related lncRNAs and all the identified genes. Only positively correlated genes with an $\mathrm{R} \geq$ 0.5 and a significant correlation $(\mathrm{P}<0.05)$ were retained. Gene Ontology term enrichment (GO) and Kyoto Encyclopedia of Genes and Genomes (KEGG) pathway analyses were performed 
using DAVID Functional Annotation Bioinformatics Microarray Analysis (https://david.ncifcrf.gov/) software.

\section{DNA Methylation Analysis}

The DNA methylation profile was measured experimentally using the Illumina Infinium HumanMethylation450 platform from the TCGA. Beta values were derived at Johns Hopkins University and the University of Southern California TCGA genome characterization center. DNA methylation values, described as beta values, were recorded for each array probe in each sample via BeadStudio software. DNA methylation beta values are continuous variables between 0 and 1, representing the ratio of the intensity of the methylated bead type to the combined locus intensity. Thus, higher beta values represent higher levels of DNA methylation, and lower beta values represent lower levels of DNA methylation.

\section{Immune Checkpoint-Related LncRNA Expression and Immune Cell Infiltration}

Molecular data for different cancer types and clinical data were downloaded from the TCGA. Moreover, the expression of immune checkpoint-related lncRNAs in different cancer tissues and normal tissues was examined using the TIMER database (https://cistrome.shinyapps.io/timer/). We also reanalyzed immune cell signature genes to estimate the abundance of immune cells (regulatory $\mathrm{T}$ cells and myeloid-derived suppressor cells) in the TIMER database. We merged the tumor gene expression with the reference immune cell data of all genes using the Bayes method, as reported in a previous study (33).

\section{Immune Checkpoint-Related LncRNA Expression and Immune Checkpoint Inhibitor Resistance}

Public datasets GSE78220, GSE91061, PRJEB23709, PRJNA482620, and phs000452.v2.p1 from GEO (http://www.ncbi.nlm.nih.gov/geo/ GEO), EGA (https://www.ebi.ac.uk/ena/browser/home) and dbGAP (https://www.ncbi.nlm.nih.gov/gap) were downloaded to investigate immune checkpoint-related lncRNA expression and immune checkpoint inhibitor resistance.

\section{RESULT}

\section{Identification of Immune Checkpoint Related LncRNA}

We renamed lncRNAs that may promote tumor immune evasion via regulation of immune checkpoint inhibitory molecules in human sarcoma "immune checkpoint-related lncRNAs" (ICLs). Sarcoma data from the TCGA were downloaded to analyze the lncRNAs positively correlated with immune checkpoint inhibitory molecules (CD274, CD80, CD86, PDCD1 LG2 and LGALS9), which accelerate the process of tumor cell immune evasion. Nine ICLs (ADAM6, C5orf58, CXCR2P1, FCGR2C, HCP5, HLA-H, NAPSB, NCF1B and NCF1C) that were positively correlated with each of the above inhibitory molecules were identified via Pearson correlation analysis $(\mathrm{r}>0.3, \mathrm{p}<5 \mathrm{E}-09)$ (Figure 1A). The relationship between the identified lncRNAs and each immune checkpoint molecule is shown in detail in Figures 1B-F.

\section{ICLs Were Overexpressed and Correlated With a Worse Prognosis in Human Cancer}

The expression of nine ICLs, ADAM6, C5orf58, CXCR2P1, FCGR2C, HCP5, HLA-H, NAPSB, NCF1B and NCF1C, was further investigated in different cancer types, including sarcoma. The results showed that the expression of nine ICLs was upregulated in different human cancer types (Figures 2 and 3). The expression of nine ICLs was further examined in sarcoma and was upregulated in sarcoma compared with the expression of the ICLs in non-cancer tissues (Figure 4A). We obtained a similar result in agreement with that observed in sarcoma in our analysis of the expression of nine ICLs in pan-cancer datasets with 9,724 patients (Figure 4B). Furthermore, the relationship between the expression of ICLs and patient prognosis was also examined. The results showed that high expression of C5orf58 indicated worse overall survival in the pan-cancer data (Figure 4C). For FCGR2C, ADAM6, HCP5, NCF1B and NCF1C, we obtained a similar result in agreement with C5orf58 (Figures 4D-H).

\section{Low DNA Methylation Modification Leads to Overexpression of ICLs}

The upstream epigenetic regulation of ICLs was further examined in human cancer. The results showed low DNA methylation at the ADAM6, HCP5 and NAPSB loci in sarcoma cancer tissues compared with that of normal tissues (Figures 5A-C). Next, the result was validated in the human pan-cancer data (Figures 5D-I). Finally, the relationship between the expression of ICLs and DNA methylation was investigated in sarcoma. For C5orf58, HLA-H and NCF1C, a negative correlation of the expression of ICLs and DNA methylation was observed in sarcoma (Figures 6A-H). These results were also validated in the human pan-cancer data.

\section{ICLs Play Vital Roles in the Negative Regulation of the Immune Response}

The guilt-by-association analysis revealed that the ICLs were involved in the regulation of key immune response processes, such as negative regulation of $\mathrm{T}$ cell proliferation, negative thymic $\mathrm{T}$ cell selection, negative regulation of interleukin-6, interferon-gamma, interleukin-17, interleukin-12, interleukin10, type I interferon, interleukin-2 and other $\mathrm{T}$ cell cytokine production, negative thymus $\mathrm{T}$ cell selection, negative regulation of $\mathrm{T}$ cell activation, negative regulation of $\mathrm{NK}$ cell-mediated cytotoxicity, negative regulation of the $\mathrm{B}$ cell receptor signaling pathway and negative regulation of B cell proliferation (Figure 7A). The potential pathways that the ICLs participated in included antigen processing and presentation, chemokine signaling pathway, Toll-like receptor signaling pathway, Rap1 signaling pathway, RIG-I-like receptor signaling pathway, cytosolic DNA-sensing pathway, TNF signaling pathway, Fc 
A

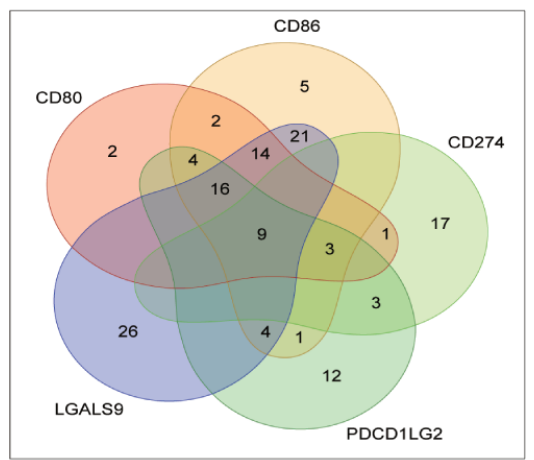

C

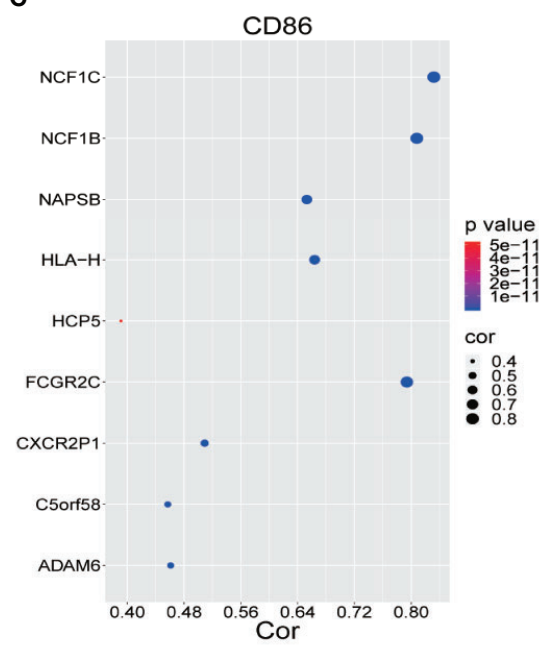

E

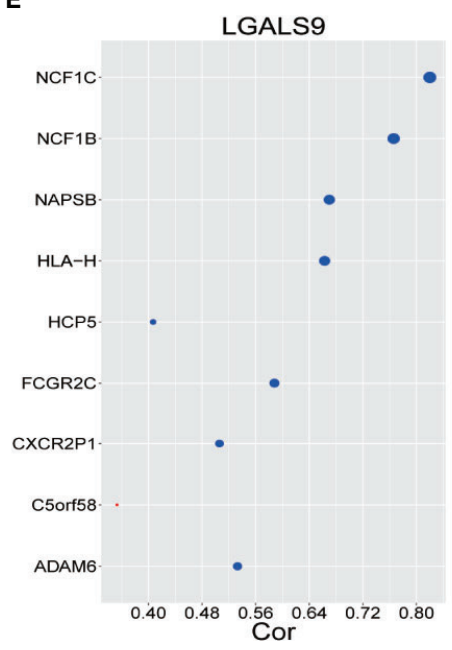

B

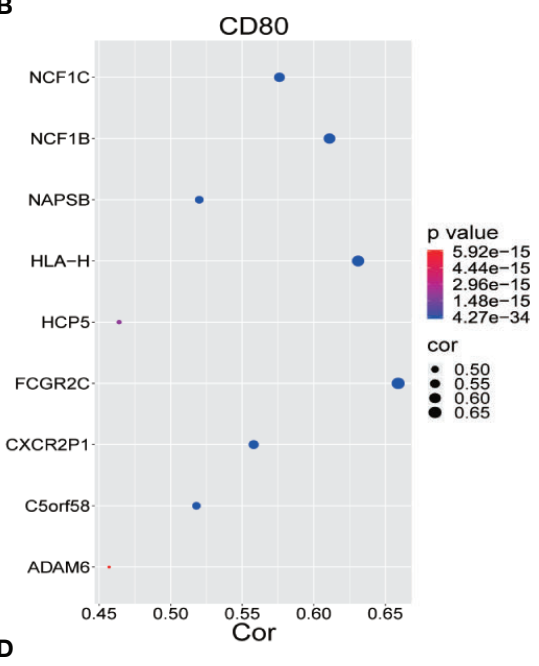

D

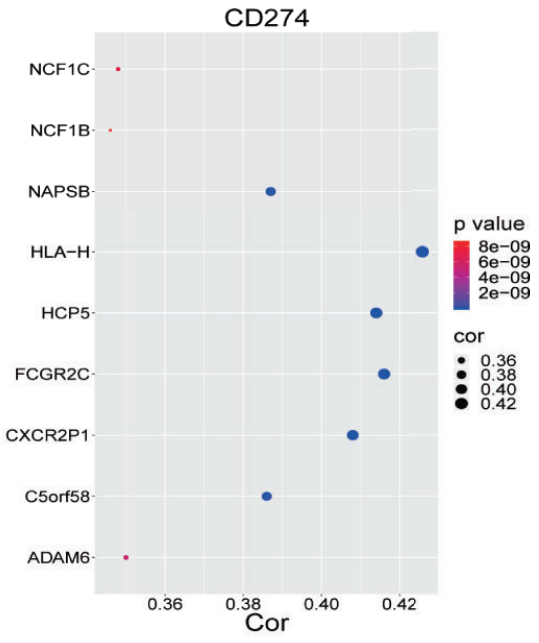

$\mathbf{F}$

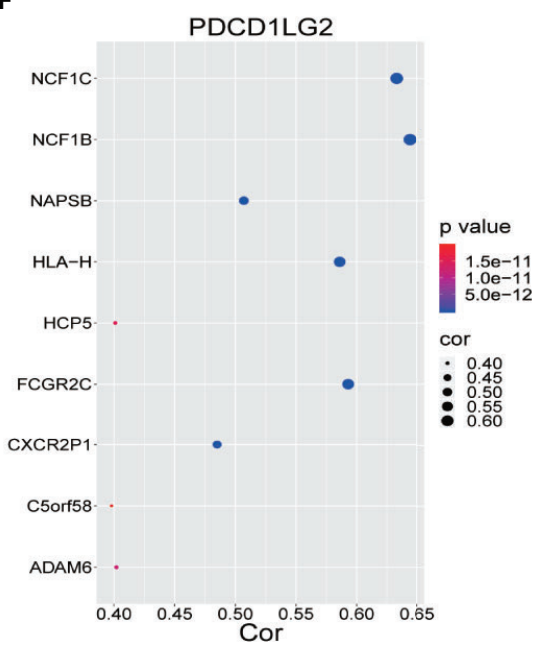

FIGURE 1 | Identification of Immune Checkpoint Related LncRNA. (A) Nine IncRNAs were identified through Pearson correlation with immune checkpoint inhibitory molecules, as shown by online Venny 2.1.0 software. (B-F) Pearson correlation between nine IncRNAs and five immune checkpoint inhibitory molecules. 

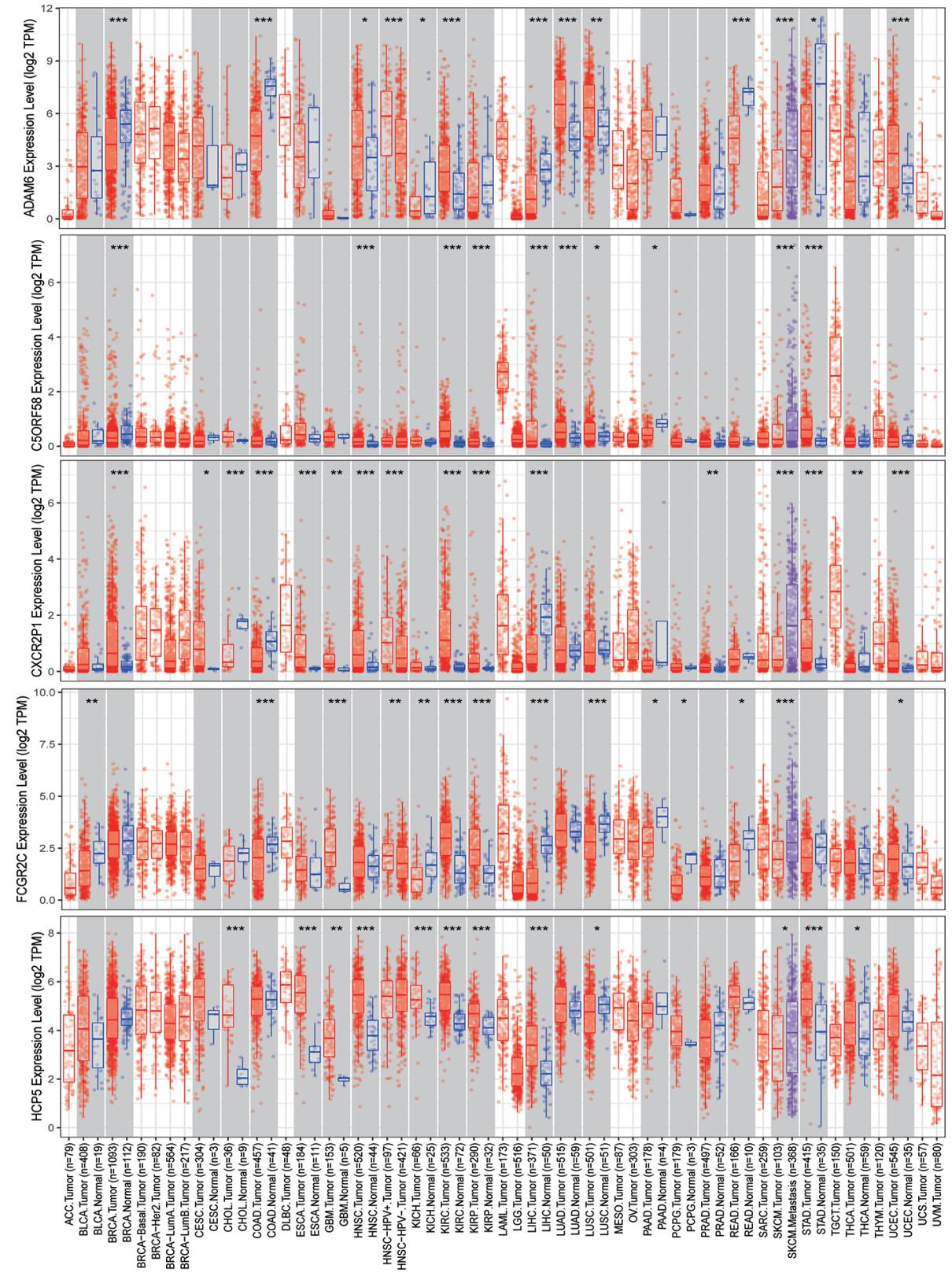

FIGURE 2 | Expression of ADAM6, C5orf58, CXCR2P1, FCGR2C, and HCP5 in different human cancer tissues and adjacent normal tissues. *P < 0.05; ${ }^{* \star} \mathrm{P}<0.01 ;{ }^{* \star *} \mathrm{P}<0.001$.

epsilon RI signaling pathway, NOD-like receptor signaling pathway, Jak-STAT signaling pathway, Fc gamma R-mediated phagocytosis, B cell receptor signaling pathway, NF-kappa B signaling pathway and $\mathrm{T}$ cell receptor signaling pathway (Figure 7B). The relationship between ICLs and immune cell infiltration in tumors was further examined in different cancers due to the finding that the ICLs negatively regulate the immune response. As expected, a high expression of ICLs was negatively correlated with $\mathrm{T}$ cell regulatory (Treg) infiltration and cancer-associated fibroblast (CAF) infiltration in 33 different cancer types (Figure 7C). Tregs and CAFs play key roles in the negative regulation of the immune response, leading to tumor progression due to immune evasion. Thus, the high expression of ICLs may lead to the failure of immunotherapy in human cancer.

\section{ICLs Confer Immune Checkpoint Inhibitor Resistance in Human Cancer}

Next, we wondered whether ICLs confer immune checkpoint inhibitor resistance in real-world studies. Public data, including data from patients treated with immune checkpoint inhibitors, were investigated to evaluate the role of ICLs on immune 


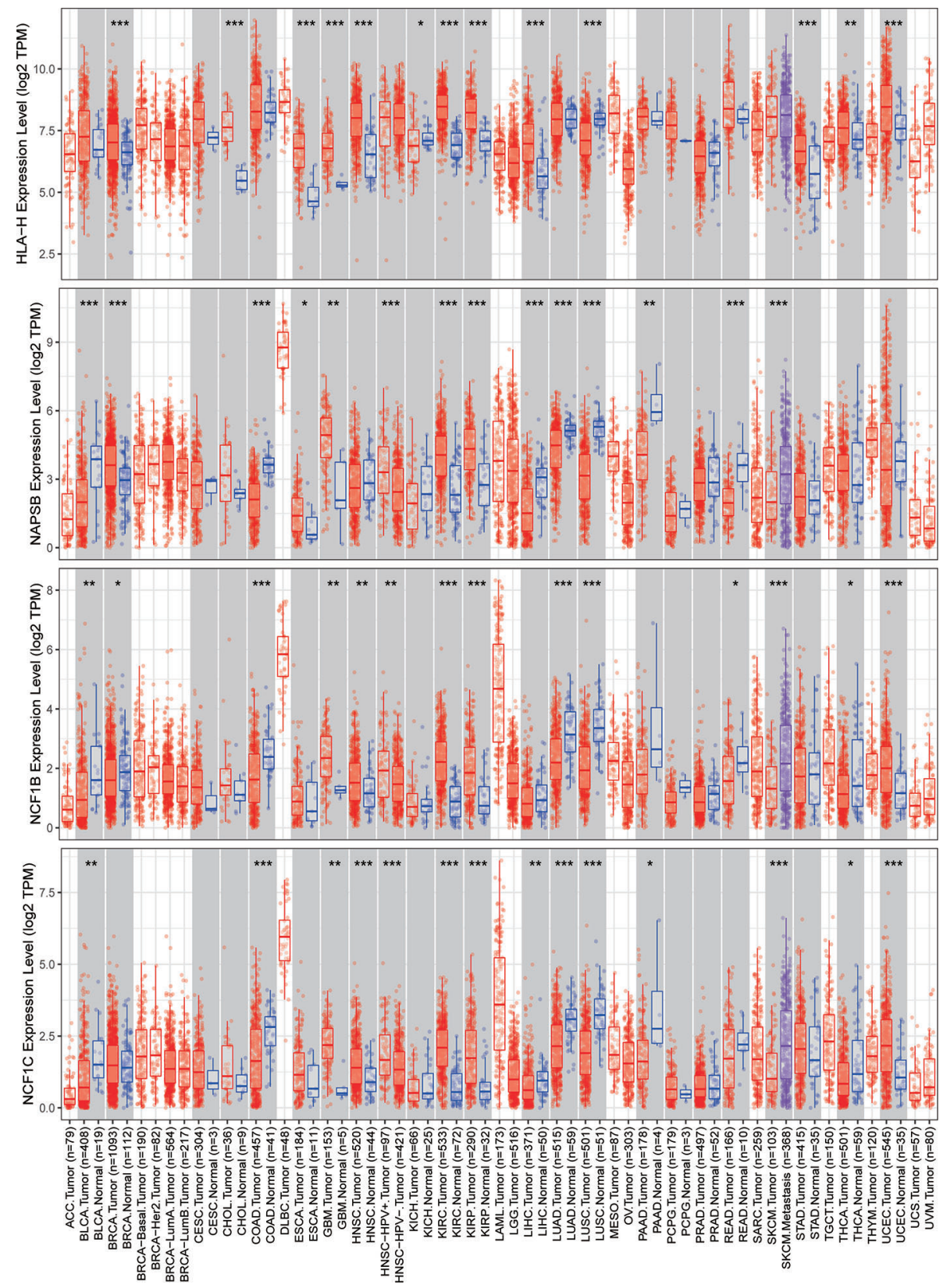

FIGURE 3 | Expression of HLA-H, NAPSB, NCF1B and NCF1C in different human cancer tissues and adjacent normal tissues. ${ }^{*} P<0.05 ;{ }^{* *} P<0.01 ;{ }^{* \star *} P<0.001$.

checkpoint inhibitors (34-37). A high expression of C5orf58 in patients with melanoma in different datasets $(34,37)$ indicated worse response to anti-PD-1/anti-CTLA-4 combined therapy, leading to worse overall survival or progression-free survival (Figures 8A, D, E). Moreover, patients with melanoma with high C5orf58 expression who received single anti-PD-1 monotherapy or anti-CTLA-4 monotherapy also showed a worse drug response, resulting in worse progression-free survival (Figures $\mathbf{8 B}, \mathbf{C})$. In addition, glioblastoma patients with a high expression of C5orf58 exhibited a disappointing response to anti-PD-1 monotherapy and worse progression-free survival (Figure 8F).

\section{DISCUSSION}

The success of adoptive cellular therapy for human cancer has resulted in tremendous enthusiasm for immunotherapy (38). Recently, immunotherapy has been approved for different 
A

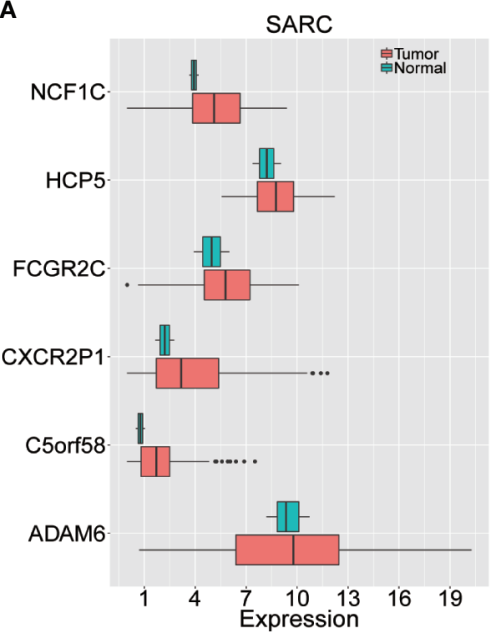

C

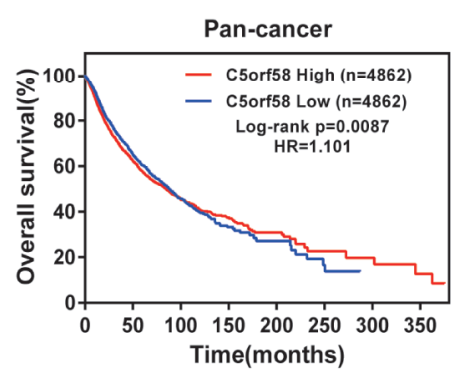

E

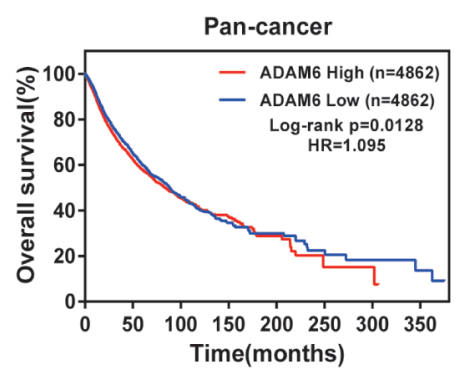

G

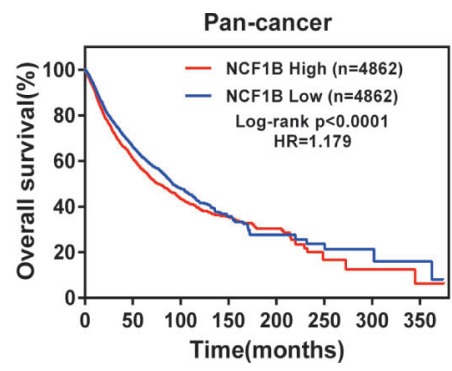

B

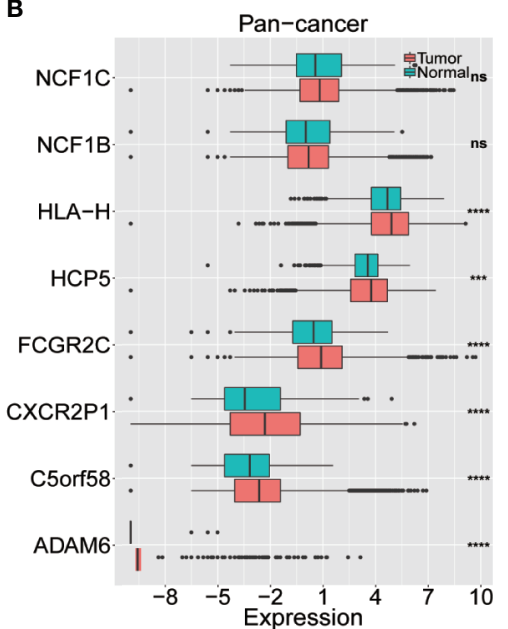

D

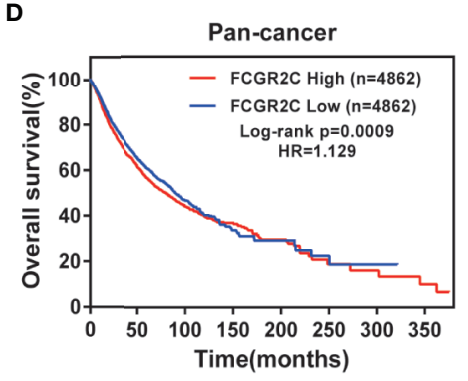

$\mathbf{F}$

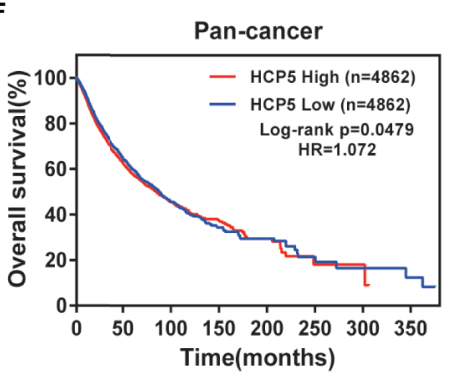

H

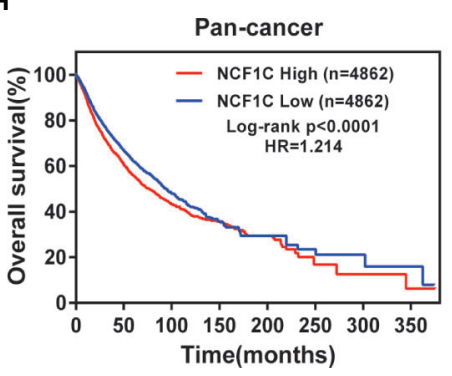

FIGURE 4 | Expression of ICLs and prognosis in human cancer. (A) Expression of ICLs in sarcoma. (B) Expression of ICLs in pancancer. (C-H) Expression of C5orf58/ FCGR2C/ADAM6/NCF1B/NCF1C and overall sunvival across cancers. (D) Expression of ICLs and overall survival in pancancer. ${ }^{\star \star \star P}<0.001 ;{ }^{\star \star \star \star} P<0.0001$; NS, No significance.

cancers, such as melanoma, prostate cancer, lymphoma, renal cell carcinoma, and breast cancer (39-53). The most successful of these strategies involve immune checkpoint inhibitors. These inhibitors increase endogenous antitumor activity and might increase the tumor immunogenicity that is induced by treatment with chemotherapy, radiotherapy and targeted therapies. These exciting outcomes have led to renewed consideration of the immunotherapy approach for sarcomas. 
A

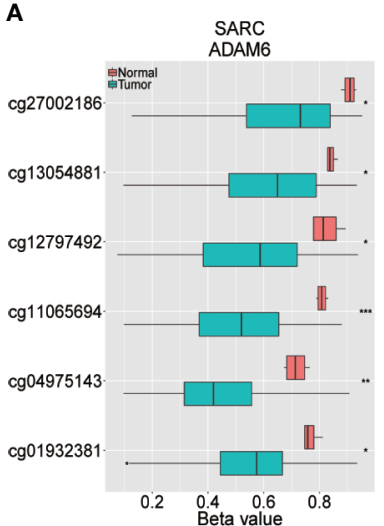

D

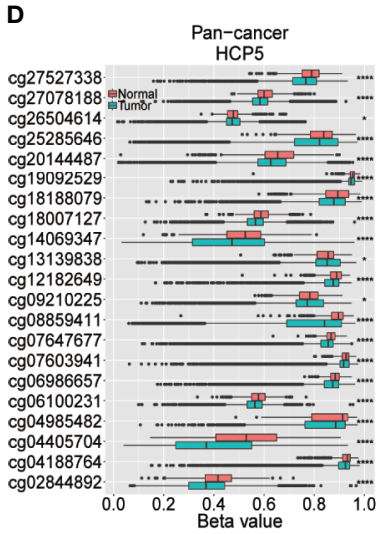

G

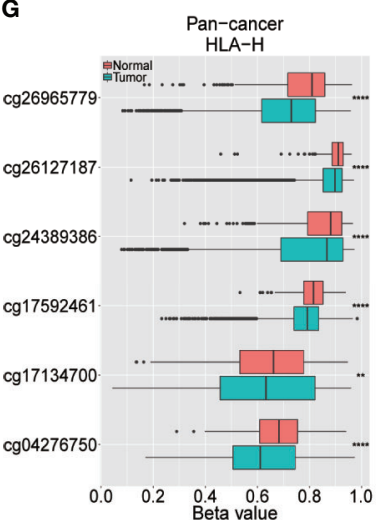

B

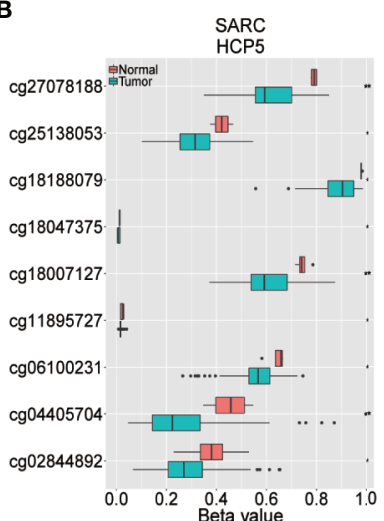

E

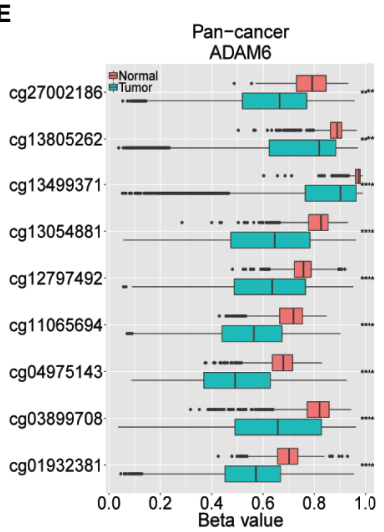

H

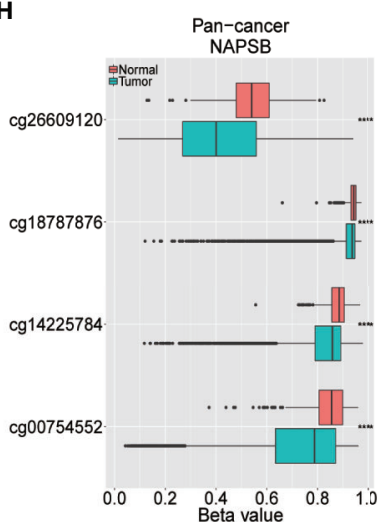

C

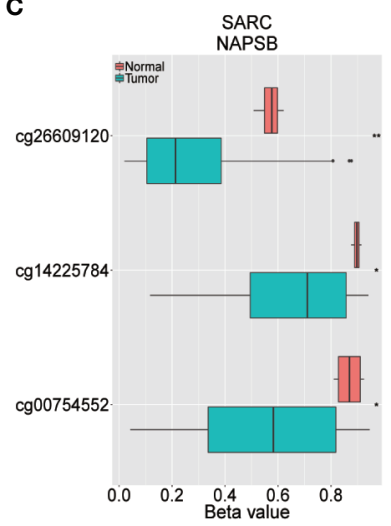

F
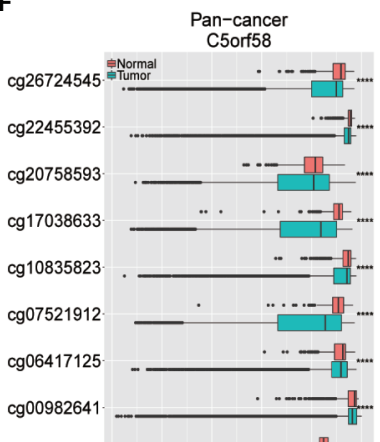

cg00787429 _-

$\begin{array}{llllll}0.0 & 0.2 & 0.4 & 0.6 & 0.8 & 1.0\end{array}$

I

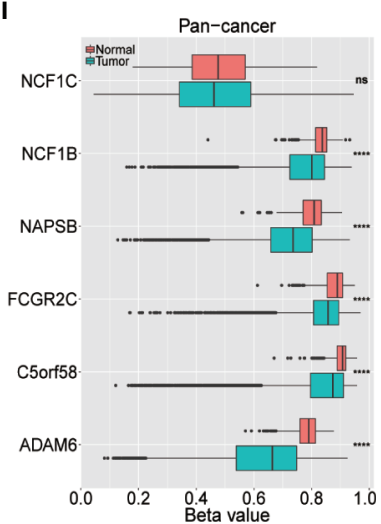

FIGURE 5 | Different methylation sites in ICLs in sarcoma and pancancer. (A-C) Different methylation sites in the ADAM6/HCP5/NAPSB/locus in sarcoma tissues and adjacent normal tissues. (D-H) Different methylation sites in the HCP5/ADAM6/C5orf58/HLA-H/NAPSB locus in pancancer tissues and adjacent normal tissues. (I) Differential methylation of IncRNAs in pancancer tissues and adjacent normal tissues. ${ }^{*} P<0.05 ;{ }^{\star \star *} P<0.01$; ${ }^{\star \star \star} P<0.001$; ${ }^{\star \star \star \star} P<0.0001$; NS, No significance.

Studies have indicated decreased overall survival in patients with higher expression of immune checkpoint proteins, such as CTLA-4 and PD-L1, suggesting that blocking immune checkpoint proteins could be therapeutically beneficial (54). The role of targetable immune checkpoint proteins such as CTLA-4 or PD-L1 in sarcomas is not yet well characterized but is a subject of active investigation. A completed phase I trial
(NCT00556881) of the anti-CTLA-4 drug ipilimumab in children and adolescents with treatment-resistant cancer included sarcomas, but no results have been reported yet. A second phase II study (NCT00140855) involving ipilimumab for patients with synovial sarcoma was stopped early due to poor accrual and no objective responses. Moreover, another study using the anti-PD-1 antibody SARC028 in patients with bone 


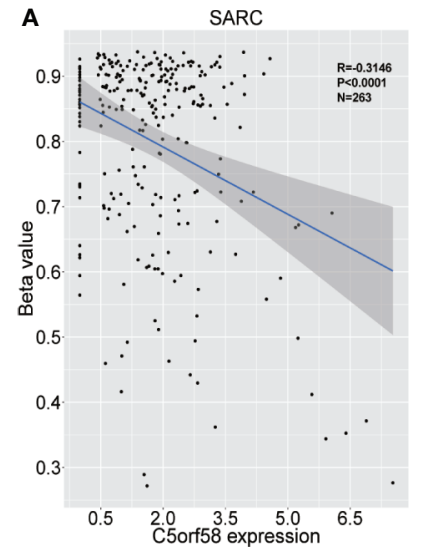

D

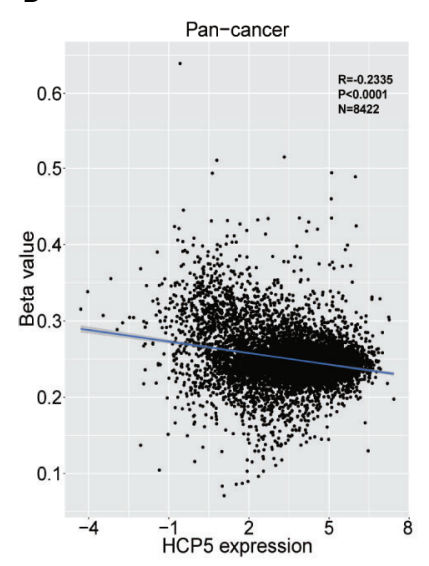

G

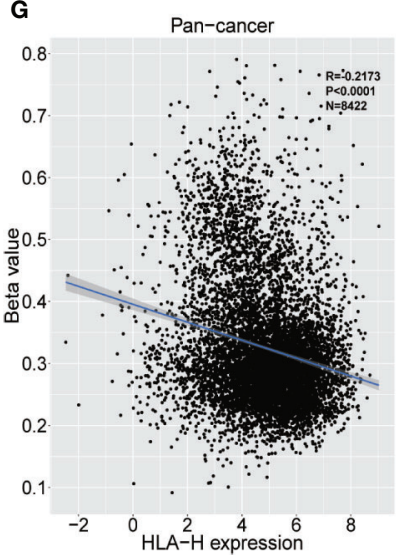

B

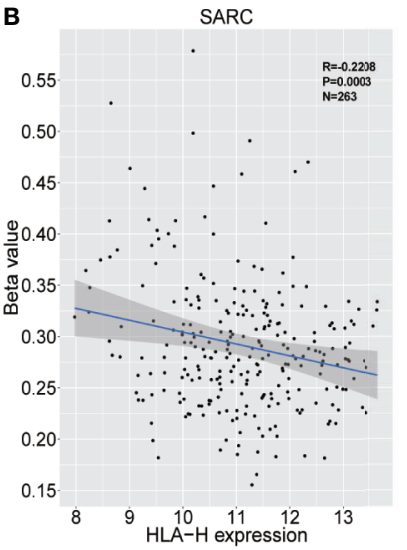

E

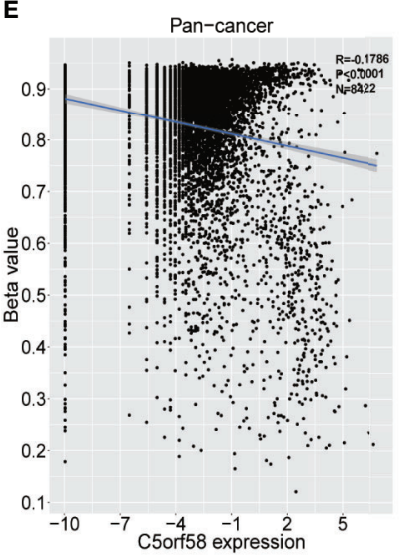

H

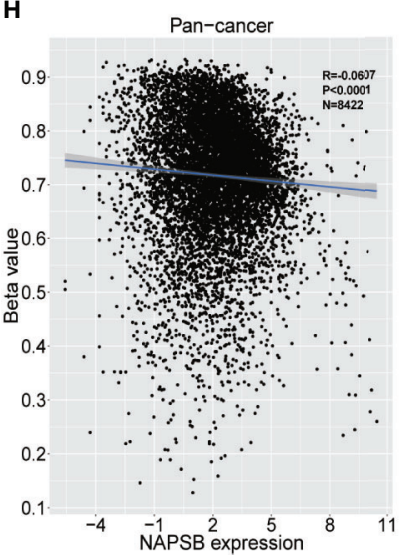

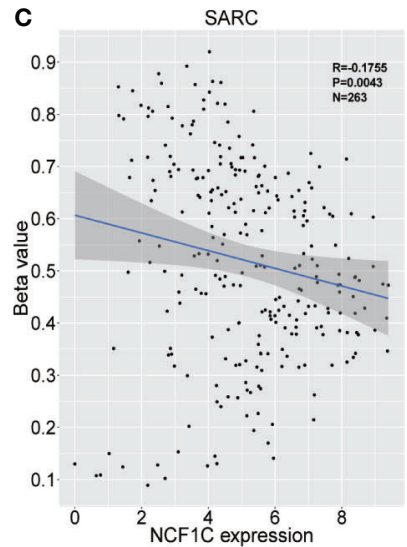

$\mathbf{F}$

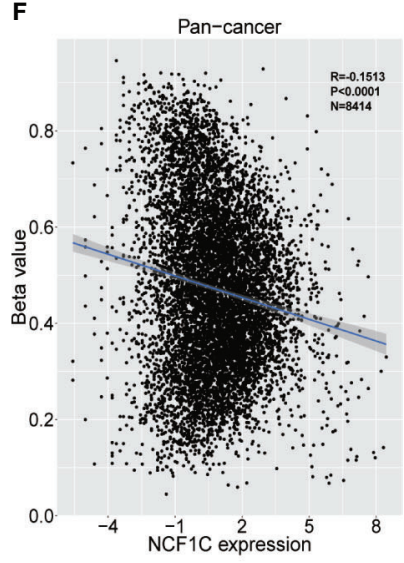

FIGURE 6 | Relationship between the expression of ICLs and DNA methylation in sarcoma and pancancer. (A-C) Correlation of C5orf58/HLA-H/NCF1C expression and DNA methylation in sarcoma. (D-H) Correlation of HCP5/C5orf58/NCF1C/HLA-H/NCF1B expression and DNA methylation across cancers.

sarcomas showed a partial response rate. Other inhibitory molecule inhibitors targeting BTLA, LAG-3, TIM3, VISTA, OX40, and CD73 have been developed. However, to date, all clinical trials of immunotherapeutic agents for sarcoma have yielded disappointing results with an insufficient patient immune response.
Primary resistance to immunotherapy may be due to an immunosuppressive environment without a preexisting antitumor response (55). To some extent, immune checkpoint inhibitory molecule overexpression may cause an immunosuppressive environment, leading to immunotherapy resistance in sarcoma. Thus, it is of great importance to 

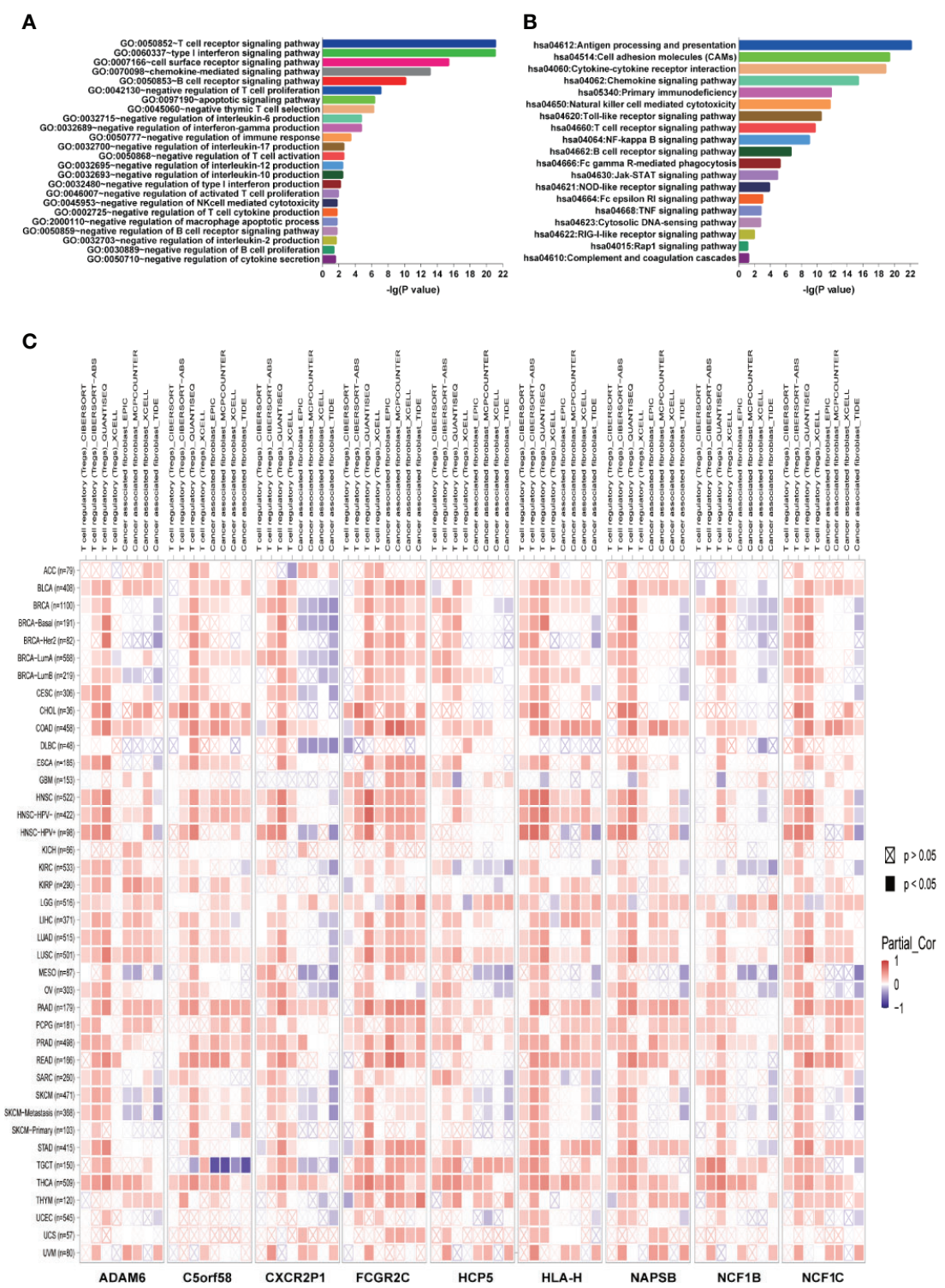

FIGURE 7 | ICLs play vital roles in the negative regulation of the immune response. (A) Geneontology term enrichment analysis for ICL-related biological processes. (B) Kyoto Encyclopedia of Genes and Genomes pathway analysis for ICL-related pathways. (C) Expression of nine ICLs and T cell regulatory/cancer-associated fibroblast infiltration in 33 human cancer types.

identify potential factors leading to immune checkpoint inhibitor resistance. In this study, we identified lncRNAs that might upregulate the expression of immune checkpoint inhibitory molecules in sarcoma. Moreover, these IncRNAs correlated with immune evasion and negative regulation of the immune response in sarcoma. High expression of these lncRNAs correlated with more suppressive immune cell infiltration and worse sensitivity to immune checkpoint inhibitors in human cancer. Thus, this study may indicate a potential cause of immunotherapy resistance issues in sarcoma that requires further investigation. Besides this study, discovery and validation of immune-associated lncRNA biomarkers correlated with immunotherapy have been performed in breast cancer, bladder cancer and lung cancer (56-58). Moreover, loss of MHC or PTEN expression was shown to be another possible mechanism in sarcoma resistance to immunotherapy after an initial positive response $(59,60)$. Thus, future clinical trials should evaluate the sarcoma tumor microenvironment and the immunological milieu that predicts the immune responsiveness of patients with sarcomas to design more effective immunotherapeutics for sarcoma.

Although initial immunotherapeutic trials for sarcoma yielded disappointing results, rational combinations of immune checkpoint inhibitors with conventional radiation or 
A

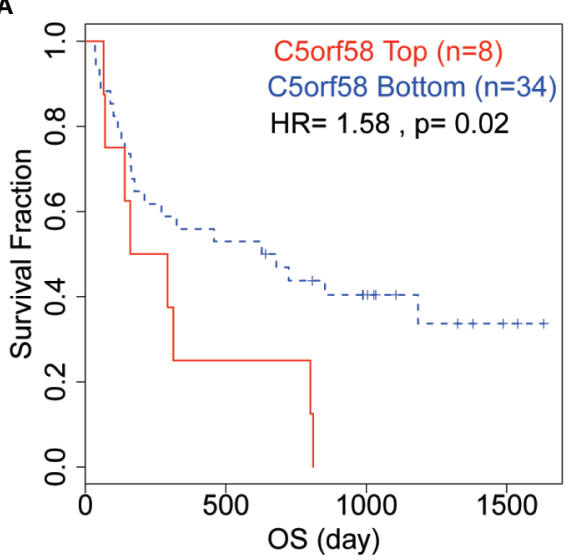

C

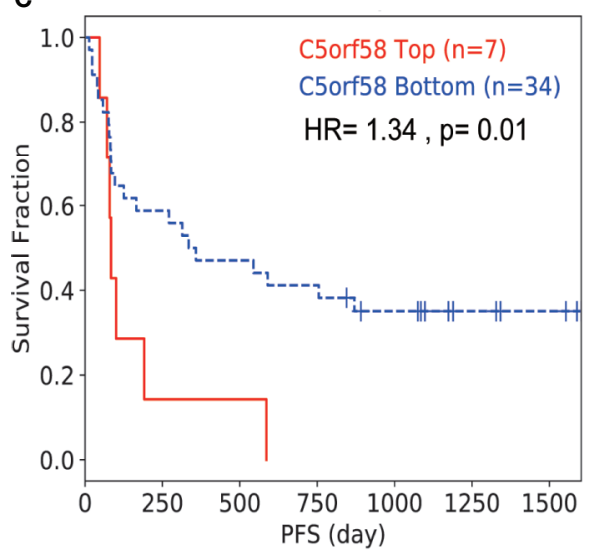

E

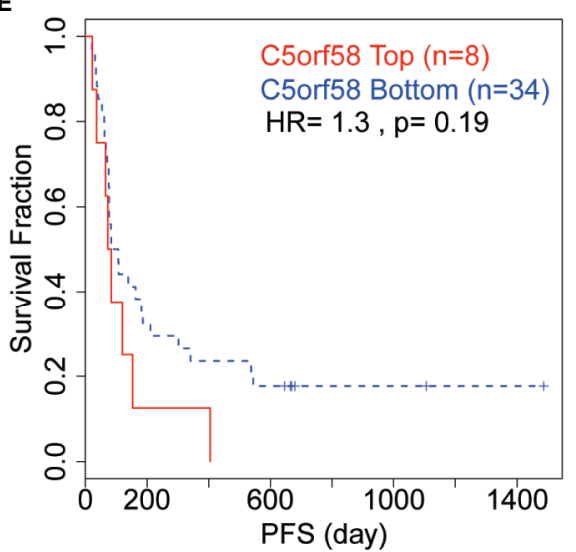

B

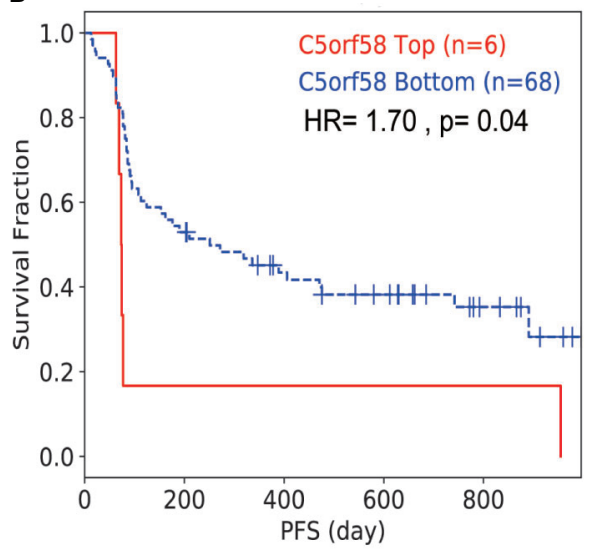

D

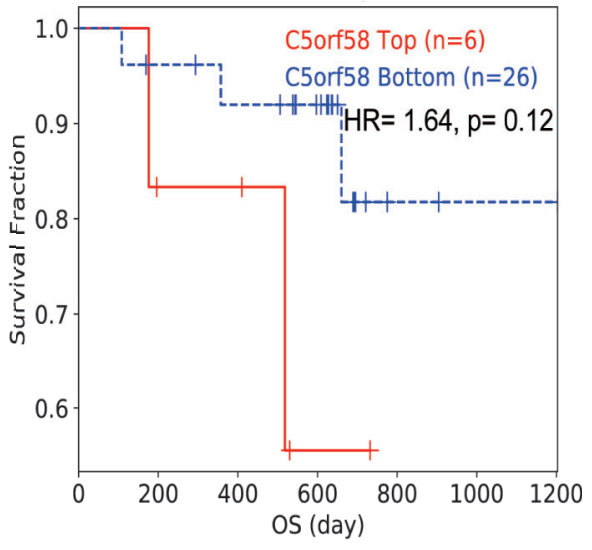

$\mathbf{F}$

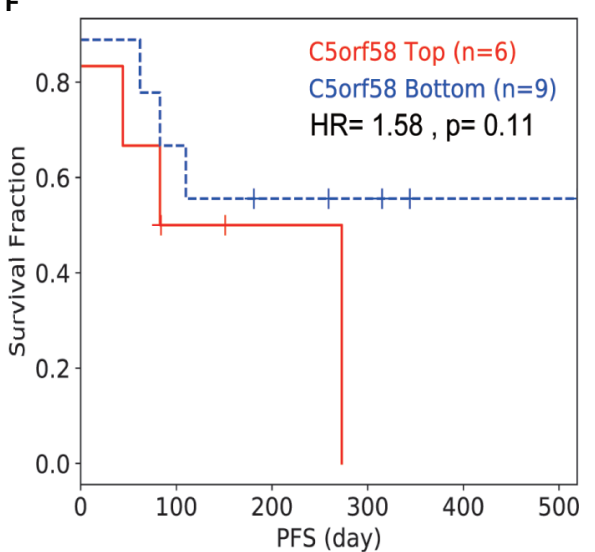

FIGURE 8 | ICLs confer immune checkpoint inhibitor resistance in human cancer. (A, D, E) Melanoma patients with high expression of C5orf58 indicated worse response to anti-PD-1/anti-CTLA-4 combined therapy and worse overall survival or progression-free survival in three datasets. (B) Melanoma patients with high expression of C5orf58 who received single anti-PD-1 monotherapy showed worse response and worse progression-free survival. (C) Melanoma patients with high expression of C5orf58 who received single anti-CTLA-4 monotherapy showed worse response and worse progression-free survival. (F) Glioblastoma patients with high expression of C5orf58 who received anti-PD-1 monotherapy showed worse response and worse progressionfree survival.

cytotoxic chemotherapy may substantially improve outcomes for patients with sarcoma in the future. Developing effective options for patients with sarcoma the greatest future challenge.
Overall, immunotherapy is a promising strategy, but the current strategies need to be refined for use in patients with sarcoma. 


\section{DATA AVAILABILITY STATEMENT}

The original contributions presented in the study are included in the article/supplementary material. Further inquiries can be directed to the corresponding author.

\section{AUTHOR CONTRIBUTIONS}

YH made substantial contributions to the project conception and design, drafted the manuscript, and gave the final approval of the

\section{REFERENCES}

1. Gamboa AC, Gronchi A, Cardona K. Soft-Tissue Sarcoma in Adults: An Update on the Current State of Histiotype-Specific Management in an Era of Personalized Medicine. CA Cancer J Clin (2020) 70(3):200-29. doi: 10.3322/ caac. 21605

2. Collignon C, Carton M, Brisse HJ, Pannier S, Gauthier A, Sarnacki S, et al. Soft Tissue Sarcoma in Children, Adolescents and Young Adults: Outcomes According to Compliance With International Initial Care Guidelines. Eur J Surg Oncol (2020) 46(7):1277-86. doi: 10.1016/j.ejso.2019.11.518

3. Ferrari A, Gasparini P, Gill J, Gorlick R. Challenges of Clinical Management of Adolescent and Young Adults With Bone and Soft Tissue Sarcoma. Cancer $J$ (2018) 24(6):301-6. doi: 10.1097/PPO.0000000000000337

4. Pandey M, Chandramohan K, Thomas G, Mathew A, Sebastian P, Somanathan T, et al. Soft Tissue Sarcoma of the Head and Neck Region in Adults. Int J Oral Maxillofac Surg (2003) 32(1):43-8. doi: 10.1054/ ijom. 2001.0218

5. Figueredo A, Bramwell VH, Bell R, Davis AM, Charette ML. Cancer Care Ontario Practice Guidelines Initiative Sarcoma Disease Site G. Adjuvant Chemotherapy Following Complete Resection of Soft Tissue Sarcoma in Adults: A Clinical Practice Guideline. Sarcoma (2002) 6(1):5-18. doi: 10.1080/ 13577140220127512

6. Bao S, Hu T, Liu J, Su J, Sun J, Ming Y, et al. Genomic Instability-Derived Plasma Extracellular Vesicle-microRNA Signature as a Minimally Invasive Predictor of Risk and Unfavorable Prognosis in Breast Cancer. J Nanobiotechnology (2021) 19(1):22. doi: 10.1186/s12951-020-00767-3

7. Blank AT, Larson BM, Shaw S, Wakefield CJ, King T, Jones KB, et al. National Comprehensive Cancer Network Guidelines Compliance of a Sarcoma Service: A Retrospective Review. World J Clin Oncol (2020) 11(6):389-96. doi: 10.5306/wjco.v11.i6.389

8. de Juan Ferre A, Alvarez Alvarez R, Casado Herraez A, Cruz Jurado J, Estival Gonzalez A, Martin-Broto J, et al. SEOM Clinical Guideline of Management of Soft-Tissue Sarcoma (2020). Clin Transl Oncol (2021) 23(5):922-30. doi: 10.1007/s12094-020-02534-0

9. Jayachandran P, Patel D, Hu J, Ragab O, Ho J, Lara K, et al. Multidisciplinary Sarcoma Tumor Board: Adolescent and Young Adult Soft Tissue SarcomaMyxoid Liposarcoma and Alveolar Soft Part Sarcoma. Chin Clin Oncol (2020) 9(5):67. doi: 10.21037/cco-20-147

10. Mesko NW, Bribriesco AC, Raymond DP. Surgical Management of Chest Wall Sarcoma. Surg Oncol Clin N Am (2020) 29(4):655-72. doi: 10.1016/ j.soc. 2020.06 .008

11. Qu C, Tian X, Ma Y, Xie X, Wang M, Dong Y, et al. Multidisciplinary Diagnosis and Treatment of Recurrent Follicular Dendritic Cell Sarcoma in Abdomen: A Case Report. Medicine (2020) 99(51):e23588. doi: 10.1097/ MD.0000000000023588

12. Tseng WW, Chen J, Patel D, Miao C, Ching A, Yang S, et al. Multidisciplinary Sarcoma Tumor Board: Retroperitoneal Liposarcoma. Chin Clin Oncol (2020) 9(2):20. doi: $10.21037 /$ cco.2020.02.08

13. Frobert P, Vaucher R, Vaz G, Gouin F, Meeus P, Delay E. The Role of Reconstructive Surgery After Soft Tissue Sarcoma Resection. Ann Chir Plast Esthet (2020) 65(5-6):394-422. doi: 10.1016/j.anplas.2020.05.009

14. D’Ambrosio L, Touati N, Blay JY, Grignani G, Flippot R, Czarnecka AM, et al. Doxorubicin Plus Dacarbazine, Doxorubicin Plus Ifosfamide, or Doxorubicin version to be published. $\mathrm{BP}$ and $\mathrm{YH}$ analyzed and interpreted the data, drafted the manuscript, and gave the final approval of the version to be published. All authors contributed to the article and approved the submitted version.

\section{ACKNOWLEDGMENTS}

The authors thank the TCGA database for the availability of the data.

Alone as a First-Line Treatment for Advanced Leiomyosarcoma: A Propensity Score Matching Analysis From the European Organization for Research and Treatment of Cancer Soft Tissue and Bone Sarcoma Group. Cancer (2020) 126 (11):2637-47. doi: 10.1002/cncr.32795

15. Brenner JC, Feng FY, Han S, Patel S, Goyal SV, Bou-Maroun LM, et al. PARP1 Inhibition as a Targeted Strategy to Treat Ewing's Sarcoma. Cancer Res (2012) 72(7):1608-13. doi: 10.1158/0008-5472.CAN-11-3648

16. Butrynski JE, D'Adamo DR, Hornick JL, Dal Cin P, Antonescu CR, Jhanwar SC, et al. Crizotinib in ALK-Rearranged Inflammatory Myofibroblastic Tumor. N Engl J Med (2010) 363(18):1727-33. doi: 10.1056/NEJMoa1007056

17. Lee HJ, Yoon C, Schmidt B, Park DJ, Zhang AY, Erkizan HV, et al. Combining PARP-1 Inhibition and Radiation in Ewing Sarcoma Results in Lethal DNA Damage. Mol Cancer Ther (2013) 12(11):2591-600. doi: 10.1158/15357163.MCT-13-0338

18. Michels S, Trautmann M, Sievers E, Kindler D, Huss S, Renner M, et al. SRC Signaling is Crucial in the Growth of Synovial Sarcoma Cells. Cancer Res (2013) 73(8):2518-28. doi: 10.1158/0008-5472.CAN-12-3023

19. Ray-Coquard I, Blay JY, Italiano A, Le Cesne A, Penel N, Zhi J, et al. Effect of the MDM2 Antagonist RG7112 on the P53 Pathway in Patients With MDM2Amplified, Well-Differentiated or Dedifferentiated Liposarcoma: An Exploratory Proof-of-Mechanism Study. Lancet Oncol (2012) 13(11):113340. doi: 10.1016/S1470-2045(12)70474-6

20. Trusolino L, Bertotti A, Comoglio PM. MET Signalling: Principles and Functions in Development, Organ Regeneration and Cancer. Nat Rev Mol Cell Biol (2010) 11(12):834-48. doi: 10.1038/nrm3012

21. Vormoor B, Schlosser YT, Blair H, Sharma A, Wilkinson S, Newell DR, et al. Sensitizing Ewing Sarcoma to Chemo- and Radiotherapy by Inhibition of the DNA-Repair Enzymes DNA Protein Kinase (DNA-PK) and poly-ADPRibose Polymerase (PARP) 1/2. Oncotarget (2017) 8(69):113418-30. doi: 10.18632/oncotarget. 21300

22. Falcone I, Conciatori F, Bazzichetto C, Ferretti G, Cognetti F, Ciuffreda L, et al. Tumor Microenvironment: Implications in Melanoma Resistance to Targeted Therapy and Immunotherapy. Cancers (2020) 12(10):2-26. doi: 10.3390/ cancers 12102870

23. Mannavola F, Mandala M, Todisco A, Sileni VC, Palla M, Minisini AM, et al. An Italian Retrospective Survey on Bone Metastasis in Melanoma: Impact of Immunotherapy and Radiotherapy on Survival. Front Oncol (2020) 10:1652. doi: $10.3389 /$ fonc. 2020.01652

24. Pala L, Conforti F, Saponara M, De Pas T, Giugliano F, Omodeo Sale E, et al. Data of Italian Cancer Centers From Two Regions With High Incidence of SARS CoV-2 Infection Provide Evidence for the Successful Management of Patients With Locally Advanced and Metastatic Melanoma Treated With Immunotherapy in the Era of COVID-19. Semin Oncol (2020) 47(5):302-4. doi: 10.1053/j.seminoncol.2020.07.010

25. Rutkowski P, Indini A, De Luca M, Merelli B, Mariuk-Jarema A, Teterycz P, et al. Body Mass Index (BMI) and Outcome of Metastatic Melanoma Patients Receiving Targeted Therapy and Immunotherapy: A Multicenter International Retrospective Study. J Immunother Cancer (2020) 8(2):1-8. doi: 10.1136/jitc-2020-001117

26. Teterycz P, Czarnecka AM, Indini A, Spalek MJ, Labianca A, Rogala P, et al. Multimodal Treatment of Advanced Mucosal Melanoma in the Era of Modern Immunotherapy. Cancers (2020) 12(11):1-15. doi: 10.3390/ cancers 12113131 
27. Maki RG, Jungbluth AA, Gnjatic S, Schwartz GK, D'Adamo DR, Keohan ML, et al. A Pilot Study of Anti-CTLA4 Antibody Ipilimumab in Patients With Synovial Sarcoma. Sarcoma (2013) 2013:168145. doi: 10.1155/2013/168145

28. Zhang Y, Zhang L, Xu Y, Wu X, Zhou Y, Mo J. Immune-Related Long Noncoding RNA Signature for Predicting Survival and Immune Checkpoint Blockade in Hepatocellular Carcinoma. J Cell Physiol (2020) 235(12):9304-16. doi: $10.1002 /$ jcp. 29730

29. Lamere AT, Li J. Inference of Gene Co-Expression Networks From Single-Cell RNA-Sequencing Data. Methods Mol Biol (2019) 1935:141-53. doi: 10.1007/ 978-1-4939-9057-3_10

30. Luo ZH, Walid AA, Xie Y, Long H, Xiao W, Xu L, et al. Construction and Analysis of a Dysregulated lncRNA-Associated ceRNA Network in a Rat Model of Temporal Lobe Epilepsy. Seizure (2019) 69:105-14. doi: 10.1016/ j.seizure.2019.04.010

31. Ruan Y, Li Y, Liu Y, Zhou J, Wang X, Zhang W. Investigation of Optimal Pathways for Preeclampsia Using Network-Based Guilt by Association Algorithm. Exp Ther Med (2019) 17(5):4139-43. doi: 10.3892/etm.2019.7410

32. Thiel D, Conrad ND, Ntini E, Peschutter RX, Siebert H, Marsico A. Identifying IncRNA-Mediated Regulatory Modules Via ChIA-PET Network Analysis. BMC Bioinformatics (2019) 20(1):292. doi: 10.1186/s12859-019-2900-8

33. Li T, Fu J, Zeng Z, Cohen D, Li J, Chen Q, et al. TIMER2.0 for Analysis of Tumor-Infiltrating Immune Cells. Nucleic Acids Res (2020) 48(W1):W50914. doi: $10.1093 /$ nar/gkaa407

34. Gide TN, Quek C, Menzies AM, Tasker AT, Shang P, Holst J, et al. Distinct Immune Cell Populations Define Response to Anti-PD-1 Monotherapy and Anti-PD-1/Anti-CTLA-4 Combined Therapy. Cancer Cell (2019) 35(2):23855.e6. doi: 10.1016/i.ccell.2019.01.003

35. Van Allen EM, Miao D, Schilling B, Shukla SA, Blank C, Zimmer L, et al. Genomic Correlates of Response to CTLA-4 Blockade in Metastatic Melanoma. Science (2015) 350(6257):207-11. doi: 10.1126/science.aad0095

36. Wang J, Sun J, Liu LN, Flies DB, Nie X, Toki M, et al. Siglec-15 as an Immune Suppressor and Potential Target for Normalization Cancer Immunotherapy. Nat Med (2019) 25(4):656-66. doi: 10.1038/s41591-019-0374-x

37. Zhao J, Chen AX, Gartrell RD, Silverman AM, Aparicio L, Chu T, et al. Immune and Genomic Correlates of Response to Anti-PD-1 Immunotherapy in Glioblastoma. Nat Med (2019) 25(3):462-9. doi: 10.1038/s41591-019-0349-y

38. Kirkwood JM, Tarhini AA, Panelli MC, Moschos SJ, Zarour HM, Butterfield LH, et al. Next Generation of Immunotherapy for Melanoma. J Clin Oncol (2008) 26(20):3445-55. doi: 10.1200/JCO.2007.14.6423

39. Ansell SM. Fundamentals of Immunology for Understanding Immunotherapy for Lymphoma. Blood Adv (2020) 4(22):5863-7. doi: 10.1182/bloodadvances.2020002537

40. Brown LC, Desai K, Zhang T, Ornstein MC. The Immunotherapy Landscape in Renal Cell Carcinoma. BioDrugs (2020) 34(6):733-48. doi: 10.1007/s40259020-00449-4

41. Chakravarty D, Huang L, Kahn M, Tewari AK. Immunotherapy for Metastatic Prostate Cancer: Current and Emerging Treatment Options. Urol Clin North Am (2020) 47(4):487-510. doi: 10.1016/j.ucl.2020.07.010

42. de Melo Gagliato D, Buzaid AC, Perez-Garcia J, Cortes J. Immunotherapy in Breast Cancer: Current Practice and Clinical Challenges. BioDrugs (2020) 34 (5):611-23. doi: 10.1007/s40259-020-00436-9

43. Di Cosimo S. Advancing Immunotherapy for Early-Stage Triple-Negative Breast Cancer. Lancet (2020) 396(10257):1046-8. doi: 10.1016/S0140-6736(20)31962-0

44. Fay EK, Graff JN. Immunotherapy in Prostate Cancer. Cancers (2020) 12 (7):1-23. doi: 10.3390/cancers 12071752

45. Franzoi MA, Romano E, Piccart M. Immunotherapy for Early Breast Cancer: Too Soon, Too Superficial, or Just Right? Ann Oncol (2020) 16(6):733-944. doi: 10.1016/j.annonc.2020.11.022

46. Graber JJ, Plato B, Mawad R, Moore DJ. Pembrolizumab Immunotherapy for Relapsed CNS Lymphoma. Leuk Lymphoma (2020) 61(7):1766-8. doi: $10.1080 / 10428194.2020 .1742903$
47. Houot R, Merryman RW, Morschhauser F. Total Immunotherapy for Hodgkin Lymphoma. Lancet Haematol (2020) 7(9):e629-30. doi: 10.1016/ S2352-3026(20)30220-9

48. Lv K, Li X, Yu H, Chen X, Zhang M, Wu X. Selection of New Immunotherapy Targets for NK/T Cell Lymphoma. Am J Trans Res (2020) 12(11):7034-47. doi: 10.1186/s13287-020-01909-y

49. Pachynski RK. A “Tail” of Immunotherapy in Metastatic Prostate Cancer. Eur Urol (2020) 78(6):831-3. doi: 10.1016/j.eururo.2020.08.040

50. Park JJ, Kellezi O, Hamasha R, Ali A, Alva AS. Immunotherapy in Metastatic Sarcomatoid Renal Cell Carcinoma: A Single Institution Experience. Cancer Treat Res Commun (2020) 25:100251. doi: 10.1016/j.ctarc.2020.100251

51. Slovin SF. Immunotherapy for Castration-Resistant Prostate Cancer: Has Its Time Arrived? Expert Opin Biol Ther (2020) 20(5):481-7. doi: 10.1080/ 14712598.2020.1735345

52. Terren I, Orrantia A, Mikelez-Alonso I, Vitalle J, Zenarruzabeitia O, Borrego F. NK Cell-Based Immunotherapy in Renal Cell Carcinoma. Cancers (2020) 12(2). doi: $10.3390 /$ cancers 12020316

53. Tokumaru Y, Joyce D, Takabe K. Current Status and Limitations of Immunotherapy for Breast Cancer. Surgery (2020) 167(3):628-30. doi: 10.1016/j.surg.2019.09.018

54. Tu L, Guan R, Yang H, Zhou Y, Hong W, Ma L, et al. Assessment of the Expression of the Immune Checkpoint Molecules PD-1, CTLA4, TIM-3 and LAG-3 Across Different Cancers in Relation to Treatment Response, TumorInfiltrating Immune Cells and Survival. Int J Cancer (2020) 147(2):423-39. doi: $10.1002 / \mathrm{ijc} .32785$

55. Saleh R, Elkord E. Acquired Resistance to Cancer Immunotherapy: Role of Tumor-Mediated Immunosuppression. Semin Cancer Biol (2020) 65:13-27. doi: 10.1016/j.semcancer.2019.07.017

56. Zhou M, Zhao H, Xu W, Bao S, Cheng L, Sun J. Discovery and Validation of Immune-Associated Long Non-Coding RNA Biomarkers Associated With Clinically Molecular Subtype and Prognosis in Diffuse Large B Cell Lymphoma. Mol Cancer (2017) 16(1):1-13. doi: 10.1186/s12943-0170580-4

57. Sun J, Zhang Z, Bao S, Yan C, Hou P, Wu N, et al. Identification of Tumor Immune Infiltration-Associated lncRNAs for Improving Prognosis and Immunotherapy Response of Patients With Non-Small Cell Lung Cancer. J Immunother Cancer (2020) 8(1):1-12. doi: 10.1136/jitc-2019-000110

58. Zhou M, Zhang Z, Bao S, Hou P, Yan C, Su J, et al. Computational Recognition of IncRNA Signature of Tumor-Infiltrating B Lymphocytes With Potential Implications in Prognosis and Immunotherapy of Bladder Cancer. Brief Bioinform (2020) 22(3):1-13. doi: 10.1093/bib/bbaa047

59. George S, Miao D, Demetri GD, Adeegbe D, Rodig SJ, Shukla S, et al. Loss of PTEN is Associated With Resistance to Anti-PD-1 Checkpoint Blockade Therapy in Metastatic Uterine Leiomyosarcoma. Immunity (2017) 46(2):197204. doi: 10.1016/j.immuni.2017.02.001

60. Morris AG, Darley RL, Bateman WJ. The Loss of Class II MHC Antigen Expression by Ras-Transformed Murine Fibroblasts Passaged as Tumours Correlates With Increased Tumorigenicity But Is Not Mediated by T Cells. Cancer Immunol Immunother (1992) 35(1):46-52. doi: 10.1007/ BF01741054

Conflict of Interest: The authors declare that the research was conducted in the absence of any commercial or financial relationships that could be construed as a potential conflict of interest.

Copyright $\odot 2021$ Pang and Hao. This is an open-access article distributed under the terms of the Creative Commons Attribution License (CC BY). The use, distribution or reproduction in other forums is permitted, provided the original author(s) and the copyright owner(s) are credited and that the original publication in this journal is cited, in accordance with accepted academic practice. No use, distribution or reproduction is permitted which does not comply with these terms. 